\title{
Ahorro Previsional Voluntario Colectivo (APVC) en Chile
}

El Ahorro Previsional Voluntario Colectivo (APVC) es una alternativa de ahorro para la vejez que permite a las organizaciones promover el ahorro previsional de sus trabajadores.

Junto con el Ahorro Previsional Voluntario (APV) y los Depósitos Convenidos (DC), el APVC es uno de los tres instrumentos que conforman el pilar de ahorro voluntario del sistema de pensiones chileno. Pese a que fue instaurado con la reforma previsional de 2008, el APVC ha sido poco utilizado.

\section{El objetivo de este artículo es describir su}

funcionamiento, presentar cifras sobre su evolución y analizar los méritos de su diseño en el contexto de las alternativas de ahorro voluntario que ofrece el sistema de pensiones chileno. El artículo concluye con una reflexión sobre las eventuales causas de su bajo nivel de adopción y explora alternativas para promoverlo.

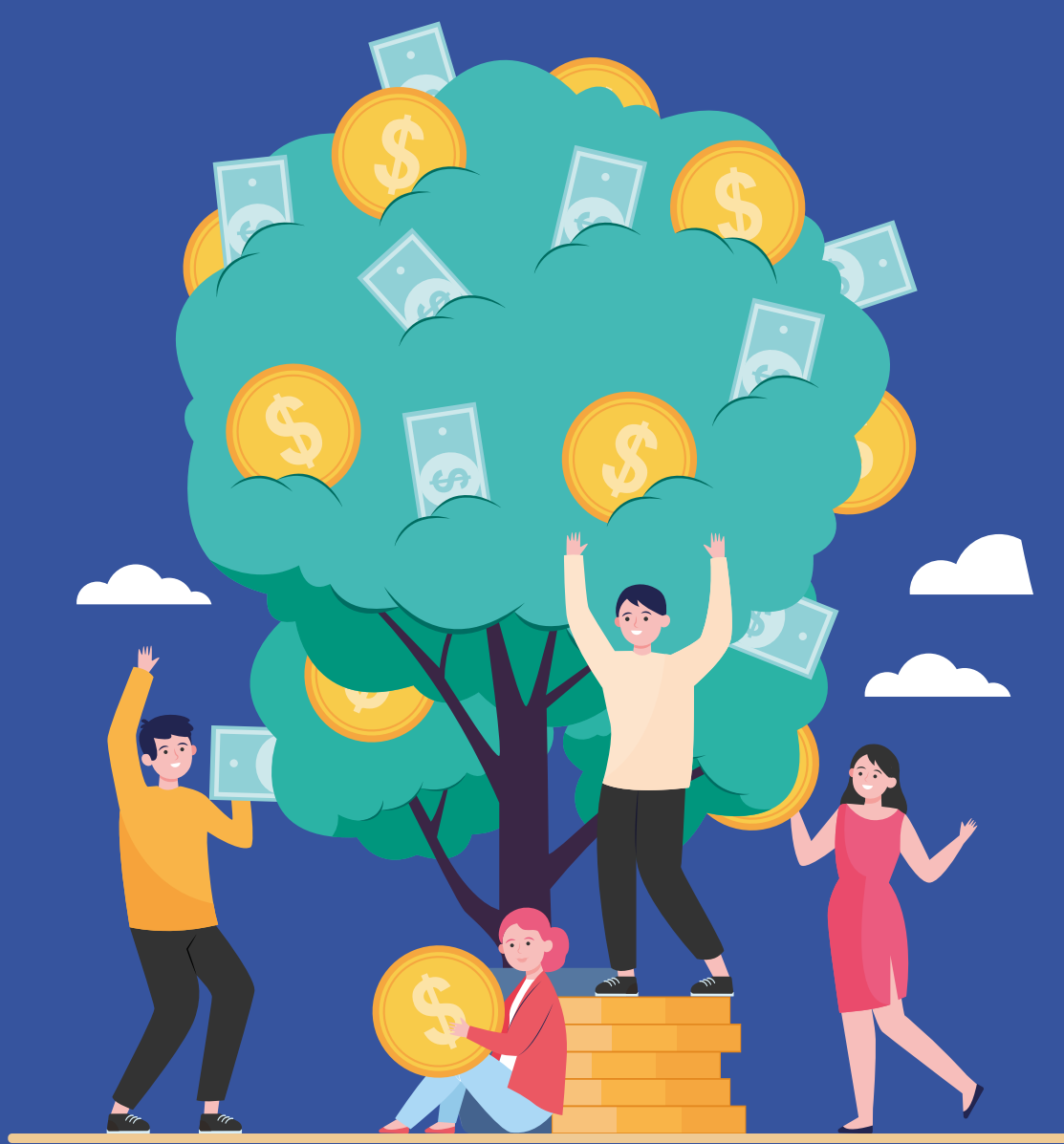

Olga Fuentes Contreras Ph.D. in Economics, Boston University, Intendenta de Regulación, Superintendencia de Pensiones

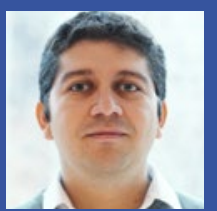

Fernando López Ph.D. in Business Administration (Finance), Washington University in St. Louis, Departamento de Gestión y Negocios, FEN-UAH

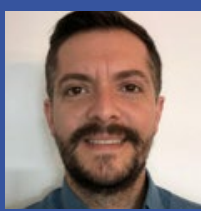

Eugenio Salvo Cifuentes Magíster en Economía, Pontificia Universidad Católica de Chile, Analista Senior, División Estudios, Superintendencia de Pensiones

\section{¿CÓMO FUNCIONA?}

I APVC es un contrato que se suscribe entre un empleador, por sí y en representación de sus trabajadores, y una institución financiera autorizada' (punto 1 de la Figura 1). Los empleadores pueden suscribir uno o más contratos de APVC con instituciones financieras y los términos y condiciones se establecen de común acuerdo entre estos. Este contrato se ofrece a los trabajadores de dicho empleador, quienes tienen la opción (no la obligación) de ahorrar para su vejez a través de los instrumentos de inversión contemplados en dicho contrato (punto 2 de la Figura 1). La regulación requiere que un mínimo de $15 \%$ de los trabajadores de la organización se encuentren adscritos al contrato. Para las organizaciones con más de 665 trabajadores, se exige un mínimo de 100 trabajadores adscritos. Un empleador puede ofrecer más de un contrato de APVC $y$, en este caso, se considera la totalidad de trabajadores adscritos a uno o más de ellos para efectos de cumplir con estos requisitos mínimos.

El APVC se basa en aportes de trabajadores y empleadores. Los aportes que estipule el empleador deben ser igualitarios para todos los trabajadores. Espe-

(1) Administradoras de Fondos de Pensiones (AFP), Bancos e Instituciones Financieras, Administradoras de Fondos Mutuos, Compañías de Seguros de Vida, Administradoras de Fondos de Inversión y Administradoras de Fondos para la Vivienda y otras autorizadas al efecto. 
cíficamente, el porcentaje de aporte que realiza el empleador, respecto del aporte del trabajador, debe ser el mismo para quienes adhieran a un mismo contrato de APVC. El empleador puede establecer un monto máximo para su aporte, el cual debe ser el mismo para todos los trabajadores.

Sin perjuicio de lo anterior, el empleador puede establecer aportes diferenciados en base a la antigüedad de los trabajadores, en la medida que todos quienes cumplan con dicho criterio de antigüedad puedan acceder a los mismos beneficios. Del mismo modo, el empleador puede obligarse a efectuar aportes para los trabajadores aunque estos no se obliguen a ello. En este caso, el empleador puede establecer condiciones distintas a las definidas para quienes si se obligaron a aportar.

Los aportes del empleador se traspasan al trabajador cuando se cumplen las condiciones establecidas en el contrato. La normativa actual exige que el máximo período para que el trabajador tenga la propiedad de los aportes realizados por el empleador sea de 24 meses por cada aporte. Este periodo de tiempo es conocido como "vesting". A los 60 meses contados desde la fecha en que el empleador debió efectuar el primer aporte, el trabajador adquiere la totalidad de los aportes del empleador y todos los aportes futuros de manera inmediata. Por su parte, los aportes del trabajador son siempre de su propiedad.

Las comisiones de los planes de APVC se acuerdan libremente entre el empleador y la Administradora o la Institución Autorizada, y solo pueden ser fijadas como porcentaje del saldo administrado. Asimismo, pueden ser diferenciadas entre contratos y en un mismo contrato pueden diferenciarse según el número de trabajadores que adhiera a este.

\section{BENEFICIOS TRIBUTARIOS}

El ahorro efectuado por la vía del APVC permite a los trabajadores acceder a los mismos beneficios tributarios que ofrece el APV a través de los regímenes tributarios A y B del Decreto Ley 3.500. Quienes optan por el Régimen A reciben una bonificación fiscal equivalente al $15 \%$ del monto ahorrado en el año, con

Figura 1

Ilustración de un contrato de Ahorro Previsional Colectivo

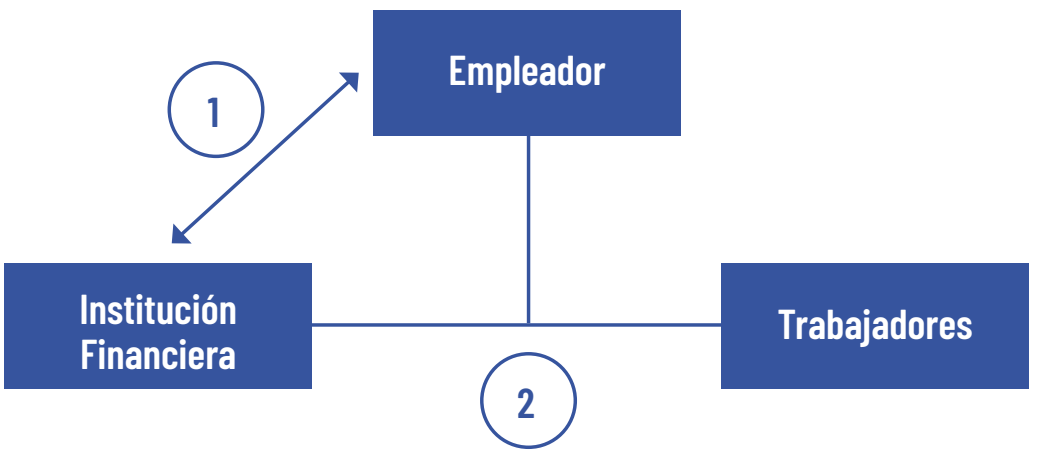

Nota: (1) representa el contrato entre el empleador y la institución financiera. (2) ilustra que cuando un trabajador suscribe un contrato de APVC, en este participa el empleador y la institución financiera.

Fuente: Elaboración de los autores.

un tope para dicho beneficio en torno a los $\$ 300.000$ anuales (6 UTM). Alternativamente, con el Régimen B los trabajadores pueden utilizar su ahorro para reducir su pago de impuestos con un tope de ahorro equivalente a UF600 anuales. En este caso, la cuantía del beneficio fiscal depende de la tasa de impuesto a la renta del trabajador $y$, por consiguiente, es conveniente para trabajadores que se encuentran afectos a una tasa (marginal) de impuesto a la renta superior al $15 \%$.

\section{VENTAJAS DEL APVC RESPECTO A OTRAS ALTERNATIVAS DE AHORRO PARA EL RETIRO}

En comparación al APV, el APVC tiene tres aspectos que lo distinguen:

1. Es un vehículo que permite al empleador tener un rol protagónico en la pensión de sus trabajadores promoviendo una cultura de ahorro, así como las instancias y herramientas que les permitan prepararse y tomar mejores decisiones previsionales.

2. El empleador puede aprovechar su escala y competencias para identificar instrumentos financieros idóneos y negociar menores comisiones con instituciones financieras, lo cual se traduce en una mayor rentabilidad para el ahorro previsional de los trabajadores. 3. El APVC permite al empleador realizar aportes en dinero que aumenten el ahorro voluntario para el retiro de los trabajadores (matching), accediendo a beneficios tributarios por ello.
Por su parte, las principales diferencias entre Depósitos Convenidos (DC) y APVC también son tres:

1. Los DC son acuerdos bilaterales entre el trabajador y empleador, que no involucran a otros trabajadores de la organización. De esta manera, el rol del empleador y su alcance es más acotado que en el caso del APVC.

2. Los DC permiten rebajar la base de impuesto a la renta con un tope de 900 UF, superando a las 600 UF permitido para la suma de APV y APVC.

3. Los DC no se pueden retirar anticipadamente como si lo permite el APVC.

\section{APVC EN CIFRAS}

EI APVC fue instaurado con la reforma previsional de 2008, a fin de complementar el ahorro previsional y mejorar las pensiones futuras de los afiliados. Sin embargo, el instrumento ha tenido baja cobertura. La Tabla 1 presenta estadísticas agregadas para el periodo 20142019. En el periodo, el número de contratos aumentó de 70 en 2014 a 92 en 2019. El número de cuentas individuales en el periodo ha aumentado de 1.155 a 1.383 en el mismo periodo. Esta última cifra representa solo un $0,02 \%$ de los 5,6 millones de los afiliados que cotizaron en el sistema a diciembre de 2019. Por último, entre 2010 y 2019 el saldo de ahorro acumulado tanto por empleadores como trabajadores paso de US\$2,4 millones a US\$4,8 millones de dólares. Esta última cifra representa solo un $0,04 \%$ del ahorro 
Tabla 1

Estadísticas agregadas APVC

\begin{tabular}{|l|c|c|c|c|c|c|}
\hline & \multirow{2}{*}{$\begin{array}{c}\text { Número de } \\
\text { contratos }\end{array}$} & $\begin{array}{c}\text { Número de } \\
\text { cuentas } \\
\text { individuales }\end{array}$ & $\begin{array}{c}\text { Saldo } \\
\text { Empleador }\end{array}$ & $\begin{array}{c}\text { Saldo } \\
\text { Trabajador } \\
\text { Régimen A }\end{array}$ & $\begin{array}{c}\text { Saldo } \\
\text { Trabajador } \\
\text { Régimen B }\end{array}$ & $\begin{array}{c}\text { Ahorro } \\
\text { acumulado }\end{array}$ \\
\cline { 4 - 7 } & & \multicolumn{3}{|c|}{ (Cifras en millones de US\$ de diciembre de 2019) } \\
\hline 2014 & 70 & 1.155 & 0,7 & 0,7 & 1,0 & 2,4 \\
\hline 2015 & 75 & 1.222 & 0,9 & 0,8 & 1,1 & 2,8 \\
\hline 2016 & 91 & 1.288 & 1,0 & 0,9 & 1,2 & 3,1 \\
\hline 2017 & 89 & 1.389 & 1,3 & 1,1 & 1,4 & 3,8 \\
\hline 2018 & 95 & 1.375 & 0,9 & 1,2 & 1,8 & 3,9 \\
\hline 2019 & 92 & 1.383 & 1,2 & 1,4 & 2,1 & 4,8 \\
\hline
\end{tabular}

Fuente: Elaboración propia con datos de la Superintendencia de Pensiones y de la Comisión para el Mercado Financiero.

Tabla 2

Participación de las AFP y Administradoras Generales de Fondos en el APVC (Promedios 2014-2019)

\begin{tabular}{|c|c|c|c|c|c|c|}
\hline & $\begin{array}{l}\text { Número de } \\
\text { contratos }\end{array}$ & $\begin{array}{l}\text { Número de } \\
\text { cuentas } \\
\text { individuales }\end{array}$ & $\begin{array}{c}\text { Saldo } \\
\text { Empleador } \\
\text { (millones de US\$) }\end{array}$ & $\begin{array}{c}\text { Saldo } \\
\text { Trabajador } \\
\text { Régimen A }\end{array}$ & $\begin{array}{c}\text { Saldo } \\
\text { Trabajador } \\
\text { Régimen B }\end{array}$ & $\begin{array}{c}\text { Ahorro } \\
\text { acumulado }\end{array}$ \\
\hline & & & \multicolumn{4}{|c|}{ (Cifras en millones de US\$ de diciembre de 2019) } \\
\hline \multicolumn{7}{|l|}{ AFPs } \\
\hline Promedio & 11 & 669 & 0,7 & 0,5 & 0,5 & 1,7 \\
\hline$\%$ del total & $13 \%$ & $51 \%$ & $65 \%$ & $53 \%$ & $37 \%$ & $50 \%$ \\
\hline \multicolumn{7}{|l|}{ Fondos Mutuos } \\
\hline Promedio & 74 & 633 & 0,3 & 0,5 & 0,9 & 1,7 \\
\hline$\%$ del total & $87 \%$ & $49 \%$ & $35 \%$ & $47 \%$ & $63 \%$ & $50 \%$ \\
\hline Promedio APVC & 85 & 1.302 & 1,0 & 1,0 & 1,4 & 3,5 \\
\hline
\end{tabular}

Fuente: Elaboración propia con datos de la Superintendencia de Pensiones y la Comisión del Mercado Financiero.

agregado en el pilar de ahorro voluntario a diciembre de 2019.

Las únicas instituciones financieras que han tenido contratos de APVC son AFPs y administradoras generales de fondos. Esto pese a que la regulación permite participar a otras instituciones financieras como bancos, compañías de seguros de vida, administradoras de fondos de inversión, corredoras de bolsa y administradoras de fondos para la vivienda.

La Tabla 2 presenta estadísticas de participación promedio para AFPs y administradoras generales de fondos para el periodo 2014-2019. Se aprecia que las
APVC, la percepción de que no contribuye a la productividad empresarial, la poca familiaridad con su funcionamiento, el esfuerzo humano y monetario asociado a su implementación o características de su diseño que lo hacen poco atractivo en el marco de la política de compensaciones a los trabajadores.

Por el lado de los sindicatos y trabajadores, el bajo interés podría explicarse por el desconocimiento sobre su funcionamiento o la preferencia por beneficios que se materializan en el corto plazo. En este caso, el APVC también compite con otros vehículos de ahorro voluntario como el APV y los DC, que se contratan a nivel individual.

La baja adopción también podría explicarse por diversos aspectos de su diseño. La restricción de un número mínimo de trabajadores adscritos en una organización, la limitada flexibilidad para diferenciar aportes del empleador entre trabajadores, el monto de los incentivos tributarios para trabajadores y empleadores, el periodo mínimo de permanencia para que los aportes pasen a la propiedad de trabajador así como la carga burocrática podrían desincentivar a los empleadores y profesionales del área de gestión de personas que podrían promover el APVC en sus organizaciones.

Por último, la evidencia internacional muestra que cuando la opción de participar en un contrato de APVC tiene como situación base la inscripción automática con libertad de salida, la tasa de adscripción es muy superior a la que se observa cuando es el trabajador quien debe enrolarse para participar (Madrian \& Shea, 2001; Choi, et al., 2004; Madrian, 2012; Clark \& Pelletier, 2018; Clark \& Pelletier, 2019; Chalmers, et al., 2020). Este es uno de los aspectos que se perfecciona en la reforma previsional y discutimos a continuación.

\section{APVC EN LA REFORMA PREVISIONAL}

les que expliquen las causas de la baja adopción del APVC en Chile. Sin embargo, existen diversas hipótesis que podrían explicarlo. Por el lado de los empleadores, la baja adopción podría explicarse por desconocimiento de la existencia del
La reforma que se encuentra actualmente en discusión en el Congreso incluye medidas para fomentar el APVC. Entre ellas se establece la opción de que los empleadores ofrezcan planes con enro- 


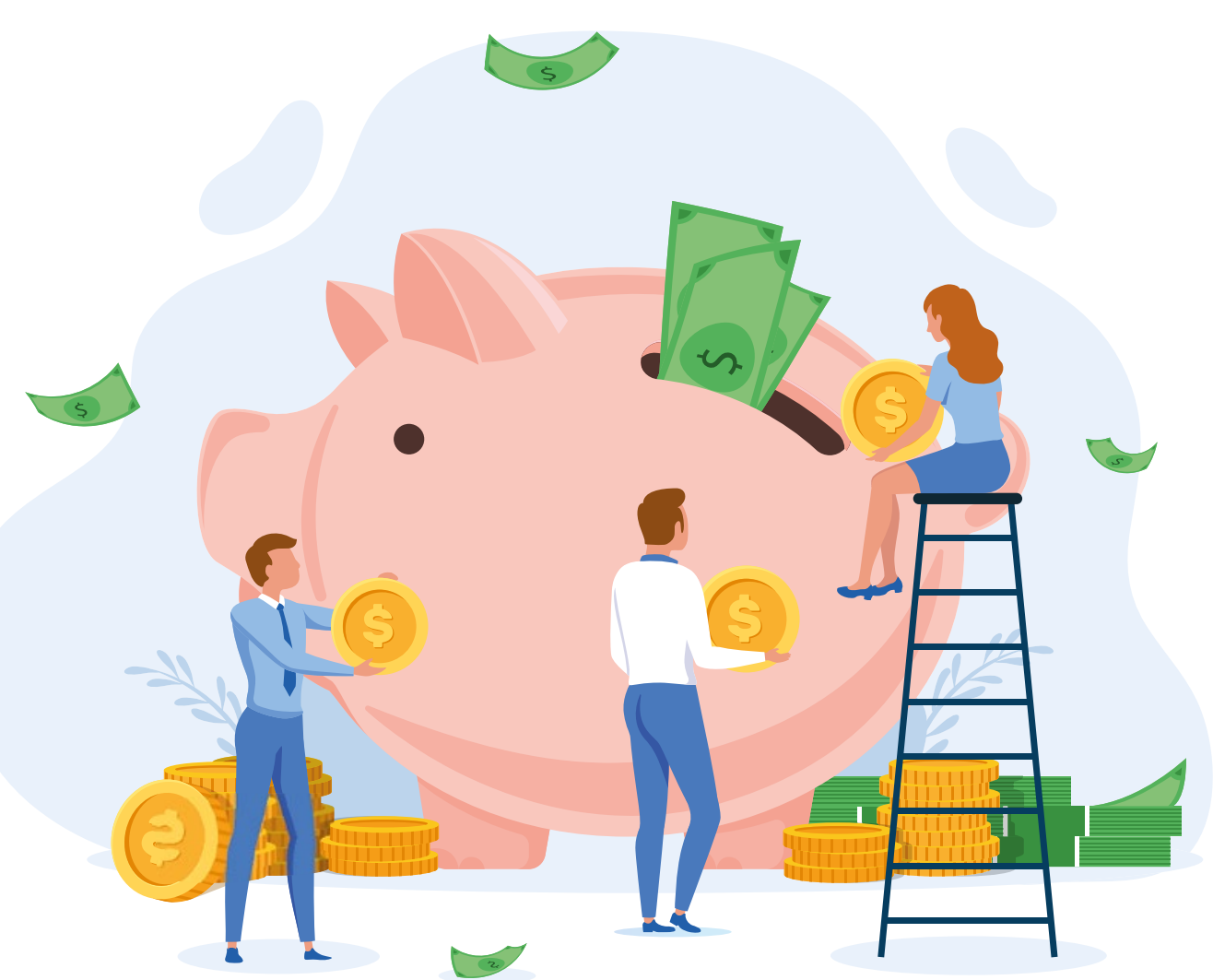

lamiento automático, de manera similar a los planes $401(k)$ que se utilizan en EEUU. En términos prácticos, bajo este esquema los trabajadores quedan automáticamente adscritos a un contrato de ahorro voluntario colectivo, a menos que expresen su voluntad de no participar. Dada la inercia de los trabajadores en sus decisiones previsionales, estos esquemas han sido exitosos en la promoción del ahorro para el retiro $y$, por consiguiente, son de especial interés para el caso chileno. Los contratos podrán también contemplar incrementos automáticos de los aportes del empleador y de sus trabajadores, así como incrementos condicionados a aumentos futuros en la remuneración.

Otras medidas de la reforma también incluyen la posibilidad de diferenciar el periodo de permanencia en el contrato de APVC y el aporte del trabajador de acuerdo al nivel de ingreso de los traba-

"El APVC es un vehículo que permite al empleador tener un rol protagónico en la pensión de sus trabajadores promoviendo una cultura de ahorro, así como las instancias y herramientas que
les permitan prepararse y tomar mejores decisiones previsionales."

jadores. La diferenciación por nivel de ingresos estará sujeta a un factor máximo de diferenciación, con el objeto de que los planes de APVC puedan beneficiar a todos los trabajadores de la empresa. De aprobarse estas medidas, los factores de diferenciación serian normados por la Superintendencia de Pensiones.

\section{COMENTARIOS FINALES}

A pesar de su potencial para contribuir a mejorar las pensiones, la cobertura del APVC ha sido baja. Son múltiples las hipótesis que podrían explicar la poca utilización del producto pero no hay estudios formales que identifiquen las principales barreras. Actualmente, la Superintendencia de Pensiones, en conjunto con el BID y RedPLAC, encargaron un estudio que busca entender las razones de su baja cobertura. Este trabajo servirá de base para elaborar una propuesta que le dé un nuevo impulso a este mecanismo de ahorro voluntario en que el empleador toma un rol protagónico en la pensión de sus trabajadores.

\section{Bibliografía}

Chalmers, J. et al., 2020. Auto-enrollment Retirement Plans for the People: Choices and Outcomes in OregonSaves. Wharton Pension Research Council Working Paper, Issue 2020-15.

Choi, J. J., Laibson, D., Madrian, B. C. \& Metrick, A., 2004. For better or for worse: Default effects and 401 (k) savings behavior. In: Perspectives on the Economics of Aging. Chicago: University of Chicago Press, pp. 81-126.

Clark, R. L. \& Pelletier, D., 2018. Impact of Defaults in Retirement Saving Plans: Public Employee Plans. NBER Working Papers 26234, National Bureau of Economic Research.

Clark, R. L. \& Pelletier, D., 2019. Does Automatic Enrollment Increase Contributions to Supplement Retirement Programs by K-12 and University Employees?. NBER Working Papers 26263, National Bureau of Economic Research.

Madrian, B. C., 2012. Matching contributions and savings outcomes: a behavioral economics perspective. In: Matching contributions for pensions: A review of international experience. Washington, D.C.: World Bank Group, pp. 289-310.

Madrian, B. C. \& Shea, D. F., 2001. The power of suggestion: Inertia in 401 (k) participation and savings behavior. The Quarterly Journal of Economics, $116(4)$, pp. $1149-1187$. 